\title{
miR-29 promoter and enhancer methylation identified by pyrosequencing in Burkitt lymhoma cells: Interplay between MYC and miR-29 regulation
}

\author{
LUCIANO MAZZOCCOLI ${ }^{1}$, MARCELA CRISTINA ROBAINA ${ }^{1}$, CARLOS E. BACCHI ${ }^{2}$, \\ SHEILA COELHO SOARES LIMA ${ }^{3}$ and CLAUDETE ESTEVES KLUMB ${ }^{1}$
}

${ }^{1}$ Laboratory of Cellular and Molecular Hemato-Oncology, Program of Molecular Hemato-Oncology,

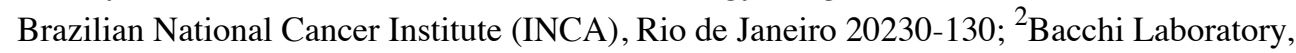

Pathology Reference Laboratory, Botucatu, São Paulo 18602-010; ${ }^{3}$ Molecular Carcinogenesis Program,

Brazilian National Cancer Institute (INCA), Rio de Janeiro 20231-050, Brazil

Received December 6, 2018; Accepted May 21, 2019

DOI: $10.3892 / o r .2019 .7183$

\begin{abstract}
Deregulation of microRNA expression plays a significant role in several cancer types including Burkitt lymphoma (BL). MicroRNA genes may be regulated through epigenetic mechanisms, such as specific histone modifications and/or DNA methylation of $\mathrm{CpG}$ islands in promoter regions, or by regions that are located next to microRNA genes. Given the regulatory role of MYC in miR-29 expression, methylation as an additional mechanism for miR-29 silencing was investigated. Methylation of miR-29a/b/c in BL tumour samples and BL cell lines (BL41 and Raji) was assessed by pyrosequencing assay. BL cells were treated with 5-aza-2'-deoxicitidine (decitabine) and evaluated for miR-29a/b/c expression and methylation status. MYC, DNMT1 and DNMT3B protein expression were accessed by western blotting. For Epstein-Barr virus (EBV) microRNA (miR)-BART6 inhibition, the cells were transiently transfected with anti-BART6-5p. BL tumour samples and BL cell lines presented miR-29a/b1 and miR-29b2/c genes methylated in $\mathrm{CpG}$ sites located in both the promoter and enhancer regions. The treatment of BL cells with decitabine reduced methylation, induced miR-29s expression and downregulated MYC protein levels in a dose-dependent manner. Notably, inhibition of EBV miR-BART6-5p combined with decitabine enhanced miR-29 expression in an EBV-BL cell line. In conclusion, the miR-29a/b1 and miR-29b2/c genes have methylated $\mathrm{CpG}$ sequences at promoter and enhancer regions that may contribute to the regulation of miR-29 expression in
\end{abstract}

Correspondence to: Professor Claudete Esteves Klumb, Laboratory of Cellular and Molecular Hemato-Oncology, Program of Molecular Hemato-Oncology, Brazilian National Cancer Institute (INCA), Praça da Cruz Vermelha, 23, $6^{\circ}$ andar ala C, Rio de Janeiro 20230-130, Brazil

E-mail: cklumb@inca.gov.br

Key words: Burkitt lymphoma, c-MYC, miR-29, methylation, DNMT
BL tumours. The present findings indicated interplay between MYC and miR-29 regulation, highlighting the potential role of EBV-miRNAs in miR-29 regulation for BL pathogenesis.

\section{Introduction}

In the last few years, increased evidence strongly indicates that altered microRNA (miRNA or miR) expression can play a significant role in cancer development and progression depending on the tissue type and specific target (1). miRNAs are small non-coding regulatory RNAs (19-25 nucleotides) that bind to specific sites of their target genes and regulate post-transcriptional gene expression (2). To date, the mechanisms regulating normal miRNA expression and triggering their deregulation in malignant diseases are poorly understood. The primary control of miRNA expression occurs at the transcriptional level. miRNA genes are frequently located in intergenic regions, being independently transcribed, or are situated within exonic and intronic regions and may share a promoter with the host gene, although intragenic miRNA-specific promoters have also been reported $(3,4)$. miRNA genes may be regulated through epigenetic mechanisms, such as specific histone modifications and/or DNA methylation of $\mathrm{CpG}$ islands in promoter regions, or by regions that are located next to miRNA genes (4). This regulation is tightly controlled and has a great impact on the establishment and maintenance of cell identity, since miRNAs, together with transcription factors, are major regulators of cell phenotype (5). Furthermore, it has been proposed that small subsets of miRNAs are capable of discriminating cell lines and tissues, reinforcing the impacts of these molecules on cell type-specific networks (6).

Burkitt lymphoma (BL) is a highly aggressive B-cell malignancy that accounts for $40 \%$ of non-Hodgkin's lymphomas in children and adolescents (7). Three clinical variants of BL, including endemic, sporadic and HIV-associated BL, have been described. Virtually all endemic BL cases are EBV-associated, whereas less than $30 \%$ of sporadic and $30-40 \%$ of AIDS-related BLs are EBV-positive (8). The tumour is characterized by the presence of chromosomal translocations, mainly $\mathrm{t}(8 ; 14)$ or, 
less frequently, $\mathrm{t}(2 ; 8)$ or $\mathrm{t}(8 ; 22)$, involving the $c-M Y C$ oncogene and immunoglobulin genes [IGH (q32), IGK (p12), and $I G L$ (q11), respectively] leading to the constitutive activity of the $c-M Y C$ promoter (9-11). Recent studies have revealed recurrent mutations in the TCF3, a direct target CCND3. In addition, CCND3 mutations were frequent in sporadic BL (38\%) and HIV-associated BL (67\%) but not endemic BL $(1.8 \%)$, indicating a distinct genetic pathogenesis among BL subtypes $(12,13)$. Emerging evidence indicates that abnormal modulation of mRNA transcription via miRNAs regulated by MYC may be a significant event in BL pathogenesis (14). In this context, a signature of miRNAs, including molecules regulated by $\mathrm{MYC}$, has been proposed to differentiate $\mathrm{BL}$ from diffuse large B-cell lymphoma (DLBCL), with miR-29b being downregulated in $\mathrm{BL}(15,16)$. This observation, together with the association between miR-29 and histone marks, that are indicative of typical enhancers and super-enhancers (5), indicates that the deregulation of miR-29 family members may play a role in BL pathogenesis. The miR-29 family has been described as a suppressor of tumours regulating multiple oncogenic pathways in diverse types of cancer (17-19). MYC also acts in the epigenetic suppression of miR-29 by inducing histone deacetylation and histone trimethylation in B-cell lymphomas (20). Additionally, DNA methylation has been associated to transcription regulation of numerous cancer-related genes including certain miRNAs such as miR-34b, miR-124a, miR-200, among others (4).

In the present study, the contribution of DNA methylation to miR-29 silencing was underlined in BL cell lines and tumour samples, targeting both promoters and enhancers using pyrosequencing quantitative assays. In addition, the current status of the interplay between MYC and miR-29 regulation was reviewed, highlighting the potential role of EBV-miRNAs in miR-29 regulation for BL pathogenesis.

\section{Materials and methods}

Cell lines and treatments. The BL cell lines: Raji $\left(\mathrm{EBV}^{+}\right)$ obtained from the American Type Culture Collection (ATCC), Daudi $\left(\mathrm{EBV}^{+}\right)$and BL41 $\left(\mathrm{EBV}^{-}\right)$kindly provided by Dr Boulanger (Greehey Children's Cancer Research Institute, The University of Texas Health Science Center, San Antonio, TX, USA), Namalwa $\left(\mathrm{EBV}^{+}\right)$kindly provided by Dr Favaro (Laboratório de Biologia Molecular e Celular, Hemocentro, Unicamp, Campinas, São Paulo, Brazil) and Ramos $\left(\mathrm{EBV}^{-}\right)$provided by Professor Andrei Thomas-Tikhonenko (Department of Pathobiology, School of Veterinary Medicine, University of Pennsylvania, Philadelphia, Pennsylvania, USA) were cultured accordingly (21). Mycoplasma contamination PCR and short tandem repeat (STR) PCR were performed to confirm that the cell lines were mycoplasma-negative and to verify their genotypes. For the experiments, cells were treated with DNMT inhibitor [5-aza-2'-deoxycytidine (decitabine); Sigma-Aldrich; Merck KGaA, Darmstadt, Germany] at $1.0,0.5,0.25$ and $0.125 \mu \mathrm{M}$ for 24 or $72 \mathrm{~h}$. The cells were collected and evaluated for DNA methylation and protein expression.

Patients. Formalin-fixed paraffin-embedded tumour samples from 10 patients with pediatric BL were selected for the analysis. The study was approved by the Brazilian National Cancer Institute Ethics Research Committee (CEP Registration no. 18/09). The diagnosis of BL was confirmed according to the criteria described by the 2008 World Health Organization (WHO) classification for hematopoietic diseases (22). Portions of these data have been previously published (23).

DNA isolation and bisulfite conversion from cell lines and tissues of patients. Genomic DNA was extracted with a QIAamp DNA FFPE Tissue kit (Qiagen, Inc.) from a tumour block fixed in $10 \%$ neutral buffered formaline and embedded in paraffin (FFPE) using sections of $10 \mu \mathrm{m}$. The average period of fixation was $24 \mathrm{~h}$ at room temperature. DNA from cells lines was extracted using the QIAamp DNA kit (Qiagen, Inc.) and quantified on a NanoDrop 1000 (Thermo Fisher Scientific, Waltham, MA, USA). Bisulfite conversion of $500 \mathrm{ng}$ of genomic DNA was performed using the EpiTect Bisulfite Kits (Qiagen, Inc.) according to the manufacturer's protocol.

Pyrosequencing assay. The previously bisulfite-converted DNA was amplified using Platinum Taq DNA Polymerase (Thermo Fisher Scientific, Inc.) in a 50- $\mu$ l final volume according to the manufacturer's protocol. The primers utilized were designed using PyroMark Assay Design Software 2.0.2 (Qiagen, Inc.): primer 1, 29b2/c gene forward, AGAAGGTAG GGTTGTAAGGA and reverse, AAATCCCCACTCTCTAAC CTATCTTTAT; primer 2, 29b2/c gene forward, TAGTAA ATATATAAGTGGGGGAAGAAGGGG and reverse, TAT CAAAACCAAAAACCTCTAAATAACC; primer 1, 29a/bl gene forward, GTTTTTTAGAGAGTTTTGGGTTGTT and reverse, CCTAAAACAAAATCCCTACAAATTTTCA. The fragments amplified with these primer sets contained 5, 6 and $5 \mathrm{CpG}$ sites, respectively. All reverse primers were biotinylated. The sequences for analysis were localized to two regions: One in the promoter of the $m i R-29 b 2 / c$ gene (Chromosome 1:207823418-207824167) and the other within the promoter flank region of the $m i R-29 b 2 / c$ gene (Chromosome 1:207823405-207824179). Another sequence for analysis was located within the enhancer of the $m i R-29 a / b 1$ gene (Chromosome 7:130897044-130897608). Two percent agarose gel electrophoresis was used to confirm DNA amplification. Biotinylated PCR products in a total volume of $40 \mu \mathrm{l}$ were immobilized on streptavidin-coated Sepharose beads (GE Healthcare). Pyrosequencing was performed using PyroGold PyroMark Q96 reagents in the PyroMark Q96 ID (both from Qiagen, Inc.). The percentage of each $\mathrm{CpG}$ site was generated automatically using the PyroMark Q96 software (version1 0.6).

miRNA extraction from BL cell lines and real-time quantitative $P C R(q R T-P C R)$. Total RNA (including the miRNA) from BL41 and Raji cells was extracted using TRIzol ${ }^{\mathrm{TM}}$ reagent (TRIzol $^{\mathrm{TM}}$; Thermo Fisher Scientific, Inc.) according to the manufacturer's instructions. The concentration and quality of the extracted material was determined with the 260/280 ratio using NanoDrop 1000 (Thermo Fisher Scientific Inc.). The miRNA expression analyses were assessed by primer-specific TaqMan ${ }^{\circledR}$ MicroRNA Reverse Transcription (Applied Biosystems; Thermo Fisher Scientific, Inc.) and hsa_miR-29a (ID 2112), hsa_miR-29b (ID 413), hsa_miR-29c (ID 587) and 
Table I. Clinical and biological features of BL patients.

\begin{tabular}{|c|c|c|c|c|c|c|}
\hline Patient & Sex/Age & Initial site of disease & Stage & LDH (U/l) & EBV (ISH) & Follow-up \\
\hline 1 & $\mathrm{~F} / 2$ & Abdomen & III & 394 & Positive & Alive \\
\hline 2 & $\mathrm{M} / 5$ & Abdomen & III & 595 & Positive & Dead \\
\hline 3 & $\mathrm{M} / 6$ & Abdomen & III & 510 & Positive & Alive \\
\hline 4 & $\mathrm{M} / 3$ & Abdomen and testicle & III & 845 & Positive & Dead \\
\hline 5 & $\mathrm{M} / 5$ & Abdomen & III & 929 & Positive & Alive \\
\hline 6 & $\mathrm{~F} / 6$ & Abdomen & III & 536 & Negative & Alive \\
\hline 7 & $\mathrm{M} / 9$ & Cervical mass & IV & 2,438 & Negative & Dead \\
\hline 8 & $\mathrm{M} / 11$ & Abdomen & III & 1,205 & Positive & Alive \\
\hline 9 & $\mathrm{M} / 5$ & Cervical mass & $\mathrm{I}$ & 453 & Positive & Alive \\
\hline 10 & $\mathrm{M} / 2$ & Abdomen & III & 621 & Positive & Alive \\
\hline
\end{tabular}

BL, Burkitt lymphoma; LDH, lactate dehydrogenase; EBV, Epstein-Barr virus; ISH, in situ hybridization.

RNU6B (NR_002752) were examined using the StepOne ${ }^{\mathrm{TM}}$ System (Applied Biosystems; Thermo Fisher Scientific, Inc.). $R N U 6 B$ was used to normalize the miRNA expression levels. Thermocycling conditions consisted of incubation at $50^{\circ} \mathrm{C}$ for $2 \mathrm{~min}$ and $95^{\circ} \mathrm{C}$ for $10 \mathrm{~min}$, followed by 40 cycles of denaturation at $95^{\circ} \mathrm{C}$ for $15 \mathrm{sec}$, and annealing and extension at $60^{\circ} \mathrm{C}$ for $1 \mathrm{~min}$. To calculate the relative expression, the $2^{-\Delta \Delta \mathrm{Cq}}$ method was applied (24). Duplicate reactions were performed in all PCR assays.

Western blotting. Western blot assays were performed as previously described (23), using the following antibodies: Anti-c-Myc (dilution 1:250; clone no. 9E10; Calbiochem; cat. no. OP10), anti-DNMT1 (dilution 1:1,000; clone no. H-300; Santa Cruz Biotechnology, Inc; cat. no. SC-13032) and DNMT3B (dilution 1:1,000, clone no. 52A1018, Imgenex; cat. no. IMG-184A). $\beta$-actin (dilution 1:1,000; clone B-6; cat. no. A5441) and HSC-70 (dilution 1:1,000; cat. no. sc-7298; both from Santa Cruz Biotechnology, Inc.) antibodies were used as loading controls. Mouse and rabbit secondary antibodies (dilution 1:10,000; cat. no. A9169) were purchased from GE Healthcare. The blots were developed using the c-Digit imaging system (LI-COR Biosciences) and analysed by Image Studio Lite version 3.1.

Transfection of an inhibitor of EBV-miR-BART6-5p. The Cy3-labbed control siRNA (Ambion; Thermo Fisher Scientific, Inc.) was used to monitor the efficiency of the transfection assay with Lipofectamine 2000 or RNAiMAX (both from Invitrogen; Thermo Fisher Scientific, Inc.). Raji cells $\left(1 \times 10^{5}\right)$ were transfected with Cy3-labeled siRNA (200 nM), washed with phosphate-buffered saline and analysed by a CyAn ADP analyzer flow cytometer and Summit v4.3 software (both from Beckman Coulter, USA). Next, Raji cells were transfected with $100 \mathrm{nM}$ of an inhibitor of BART6-5p antagomir (cat. no. 4464084) or the mimic negative control (cat. no. 4464058) (Life Technologies; Thermo Fisher Scientific, Inc.) utilizing the method derived from Mazzoccoli et al (21). Then, the cells were treated with decitabine $(1.0 \mu \mathrm{M})$ and after $72 \mathrm{~h}$, the cells were harvested, and miRNA was extracted and quantified as aforementioned.
Statistical analysis. Comparisons among groups were performed using Kruskal-Wallis and Dunnett's post hoc test. P-values $<0.05$ were considered to indicate a statistically significant difference, and analysed using GraphPad Prism software (PRISM 5.0; GraphPad Software Inc.).

\section{Results}

miR-29a/bl and miR-29b2/c genes are epigenetically silenced by methylation at promoter and enhancer regions in BL cells. To confirm previous research that revealed methylation in miR-29a/b1 and miR-29b2/c by MSP assays in BL cell lines, the miR-29 promoter and enhancer regions were explored using the pyrosequencing method for investigation of BL tumour samples. Data of BL patients are described in Table I. Quantitative pyrosequencing methylation analysis of five $\mathrm{CpG}$ sites for miR-29a/b1 enhancer region and six $\mathrm{CpG}$ sites for miR-29b2/c promoter region demonstrated high levels of DNA methylation in 10/10 and $7 / 10 \mathrm{BL}$ tumour samples, respectively as depicted (Fig. 1). The mean of methylation levels in CpG sites was $92.8 \%$ in miR-29a/b1 enhancer and $64 \%$ in miR-29b2/c promoter in BL tumour samples. Additionally, the high methylation level observed in BL tumour samples could explain the low miR-29s levels reported previously by our group (23). In Raji and BL41 cell lines the results also revealed mean methylation levels of 96 and $99 \%$ in $\mathrm{CpG}$ sites located in miR-29a/b1 enhancer and 89 and $91 \%$ in miR-29b2/c promoter flanking regions, respectively (Fig. S1A and C). Notably, the mir-29b2/c promoter from Raji cells revealed lower mean methylation levels (63\%) than in BL41 cells (92\%) at six evaluated $\mathrm{CpG}$ sites (Fig. S1B). Thus, using BL tumour tissues analysed by pyrosequencing, it was concluded that silencing by methylation can work together with other mechanisms to potentiate the suppression of miR-29 in BL cells (20).

The treatment of BL cells with DNA-demethylating agent decitabine reverses methylation and induces miR-29 expression. Since tumour suppressive miRNAs are inactivated in cancer cells by aberrant DNA methylation at promoter regions, resulting in suppression, the effects of demethylating agent decitabine was investigated on the miR-29 methylation 
A
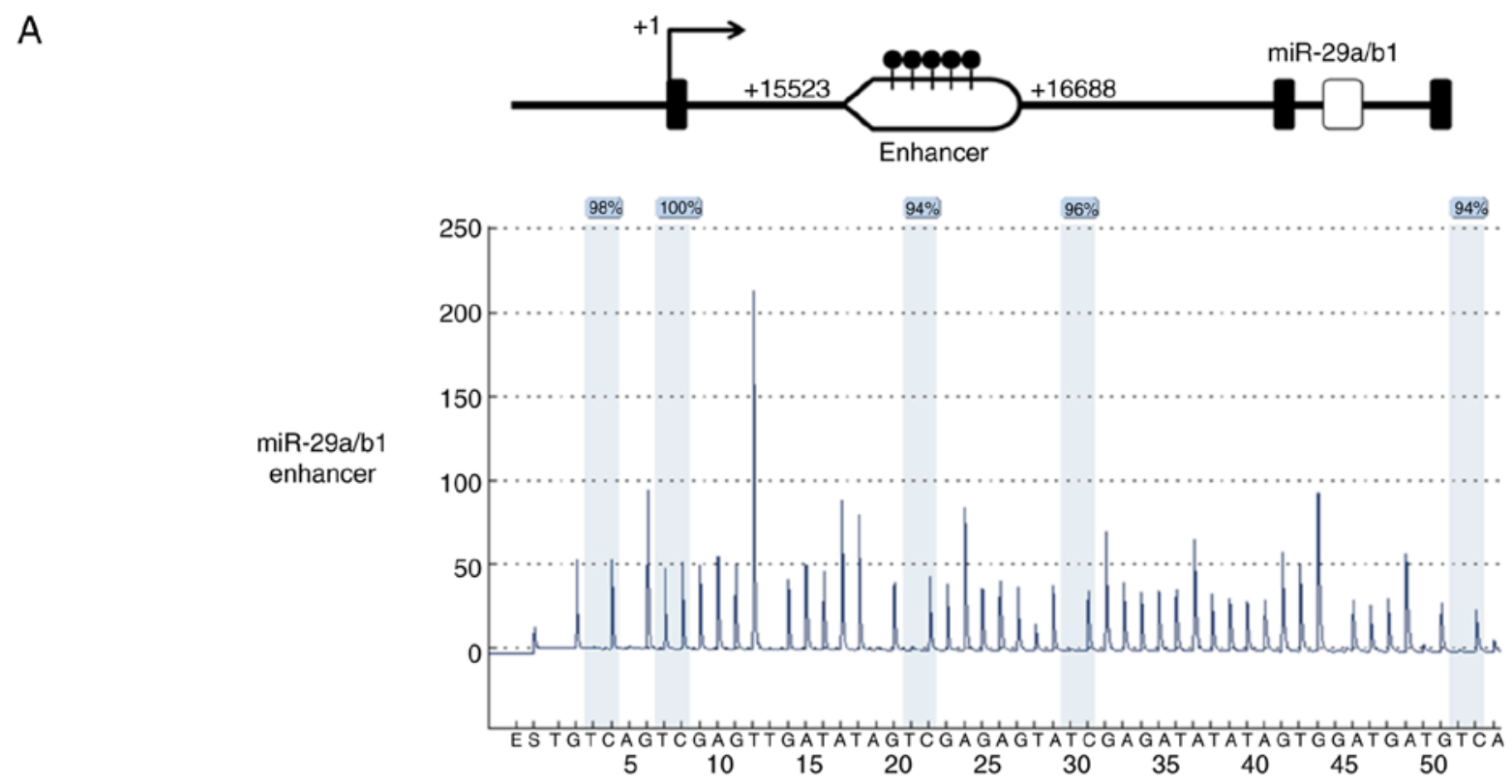

\begin{tabular}{|c|c|c|c|c|c|}
\hline BL tumor samples (10/10) & CpG 1 & CpG 2 & CpG 3 & CpG 4 & CpG 5 \\
\hline \% of methylation (median and SD) & $97.1 \%$ & $97.9 \%$ & $91.3 \%$ & $95.3 \%$ & $92.9 \%$ \\
& \pm 0.73 & \pm 2.28 & \pm 1.25 & \pm 1.7 & \pm 1.19 \\
\hline
\end{tabular}

B
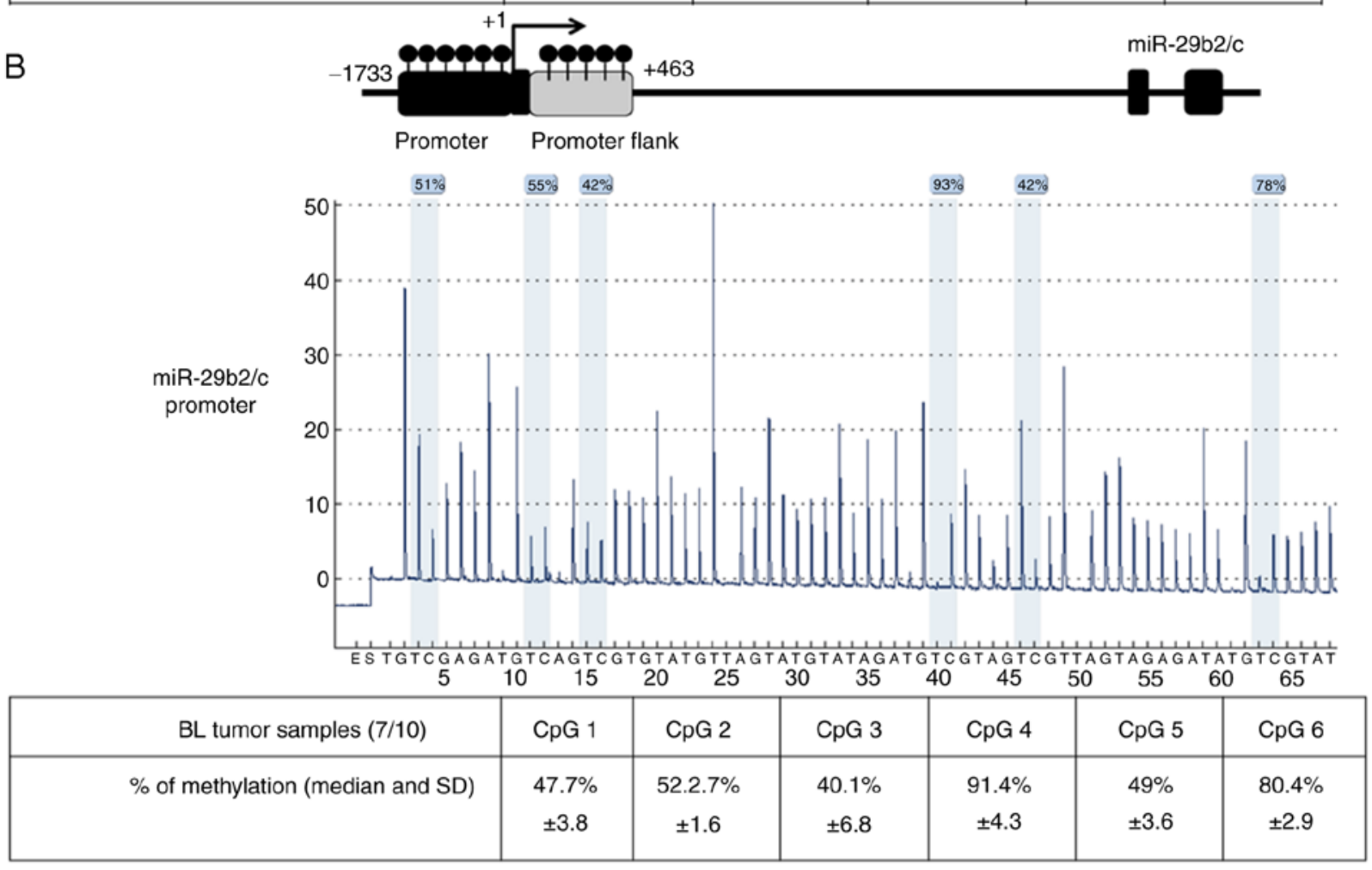

Figure 1. Quantitative pyrosequencing analysis reveals methylation on miR-29a/b1 enhancer and miR-29b2/c promoter regions in BL tumour samples. The genomic locations of pyrosequencing assays and representative pyrograms in BL tumour samples are depicted. (A) miR-29a/b1 enhancer region, (B) miR-29b2/c promoter region. The percentage of methylation at each $\mathrm{CpG}$ site is represented in shaded areas. Mean and standard deviation are presented in the box at the bottom of each pyrogram. BL, Burkitt lymphoma.

and expression levels in BL cells. After $24 \mathrm{~h}$ with $1.0 \mu \mathrm{M}$ decitabine, the miR-29 promoter and enhancer regions were demethylated in both BL cell lines. In BL41 cells, the mean of cells that were methylated in CpG sites dropped $22 \%$ in the miR-29a/b1 enhancer, $21 \%$ in the miR-29b2/c promoter and
$56 \%$ in the miR-29b2/c promoter flank in comparison to the control. As for Raji cells, the mean of methylation levels in CpG sites dropped $40 \%$ in the miR-29a/b1 enhancer, $21 \%$ in the miR-29b2/c promoter and $20 \%$ in the miR-29b2/c promoter flank in comparison to the control (Fig. S1). Similar results 
A

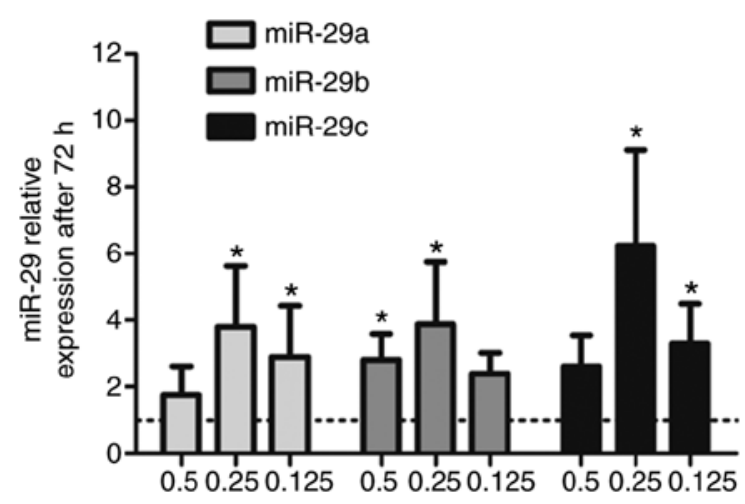

B

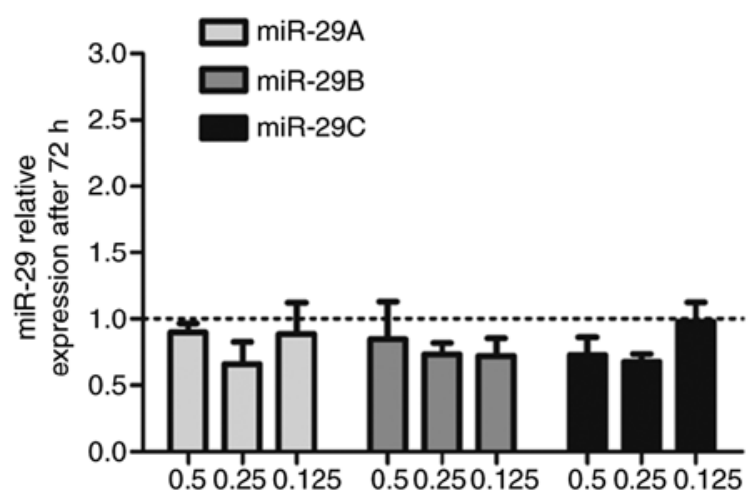

Figure 2. The expression of miR-29s in BL cells is upregulated by decitabine treatment. Quantitative PCR of miR-29a/b1 and miR-29b2/c in the BL41 and Raji cell lines. The miR-29 expression levels were analysed after treatment with low doses of decitabine. (A) BL41 and (B) Raji cells were treated with decitabine $(0.5,0.25$ and $0.125 \mu \mathrm{M})$ and evaluated after $72 \mathrm{~h}$ for $\mathrm{miR}-29 \mathrm{a} / \mathrm{b} / \mathrm{c}$ expression. The data represent the mean of three independent experiments and respective standard deviation, whereas the dotted line represents untreated cells (negative control). Statistical analysis was performed using Kruskal-Wallis and Dunnett's post hoc test. ${ }^{*} \mathrm{P}<0.05$. Decitabine, 5-aza-2'-deoxycytidine.

were observed at $72 \mathrm{~h}$, even using lower decitabine concentrations (Fig. S2). In addition, the miR-29 expression levels were upregulated by decitabine treatments $(0.5,0.25$ and $0.125 \mu \mathrm{M})$ at $72 \mathrm{~h}$ in BL41 cells (Fig. 2A) but not in Raji cells (Fig. 2B). Similar results only for the high-decitabine concentration were reported previously by our group using a non-quantitative methylation-specific PCR assay (MSP) (21).

DNA methyltransferase inhibitor, decitabine, downregulates MYC protein levels in a dose-dependent manner. Given that MYC is involved in miR-29 regulation, we further evaluated the effect of decitabine on MYC protein expression. Upon 24 and $72 \mathrm{~h}$ of decitabine treatment $(1.0 \mu \mathrm{M})$, MYC expression levels were reduced in BL41 cells (Fig. 3A). Notwithstanding the variations observed on MYC expression levels in the control (without treatment of decitabine), these variations were expected due the prolonged cell culture ( 24 to $72 \mathrm{~h}$ ), since the medium was not replaced during the assay. The cell cycle status and the concentration of fetal bovine serum, for example, may contribute to the stabilization of mRNA and protein levels. In addition, a slight decrease in the level of $\beta$-actin was detected in the presence of decitabine. However, this variation did not have impact on MYC expression analysis (Fig. 3A). A similar result was observed in Raji cells at $24 \mathrm{~h}$ after decitabine treatment (Fig. 3B). These data indicated that demethylation induced by decitabine may affect other genes or miRNAs that target the MYC regulatory network (25). However, lower doses of decitabine reduced DNMT1 expression in BL41 and Raji cells with no effects on MYC protein levels (Fig. 3C and D).

EBV-miR-BART6-5p inhibition modulates miR-29 expression in BL cells treated with decitabine. It has been reported that EBV regulates the expression of non-coding RNA in gastric carcinoma (GC). The methylation of both viral and host DNA is one of the major mechanisms involved in EBV-associated GC tumour development (26). These studies indicate that EBV infection alters the host miRNA profile. In our study, despite the demethylation of miR-29 promoter $\mathrm{CpG}$ sites observed in Raji, an EBV-positive cell line, an increase in the expression levels was not detected (Fig. 2B). Therefore, whether the observed lack of miR-29 expression could be related to the expression of EBV-miRNAs was investigated since it has been reported that an EBV-miRNA, miR-BART6-5p, suppresses Dicer expression by binding to four target sites that are present within the 3'-UTR of human Dicer (27). Then, miR-BART6-5p expression was silenced and the effect of decitabine on miR-29 expression levels was examined. The transfection efficiency assay was confirmed in the Raji cell line using Cy3-labeled control siRNA as revealed in Fig. S3.

Suppression of miR-BART6-5p by antagomir followed by decitabine treatment was associated with an increase of $\mathrm{miR} 29 \mathrm{a} / \mathrm{b} / \mathrm{c}$ expression levels in comparison to only antagomir (Fig. 4A). There was no difference between the mimic negative control and non-transfected cells regarding miR-29 expression (data not shown). In addition, the basal miR-29 expression levels in the BL41 cell line were increased in comparison to Raji cells, supporting the hypothesis of a differential miR-29 regulatory mechanism between EBV-positive and EBV-negative BL cells (Fig. 4B). The analysis of additional BL cell lines (EBV-positive and negative) indicated the same differential expression levels of miR-29a and miR-29c (Fig. S4).

\section{Discussion}

Recent studies have revealed the numerous roles of miRNAs underscoring the biological significance of their alterations in cancer development $(1,28)$. The miR-29 family of miRNAs has been identified to function as a group of tumour suppressors $(29,30)$ in several cancer types via inhibition of targets related to proliferation, migration and invasion $(31,32)$. In addition, the miR-29 family members (miR-29a/b/c) are also termed 'epi-miRs' due to their role in the regulation of epigenetic modifiers such as enzymes involved in DNA methylation and demethylation [DNA methyltransferases [DNMTs] and ten-eleven translocation (TET) enzymes] (4). Recently, our group demonstrated that the transfection of miR-29s modulated DNA methyltransferase 3B in BL cells. Moreover, overexpression of $\mathrm{miR}-29 \mathrm{a} / \mathrm{b} / \mathrm{c}$ resulted in the inhibition of other targets 

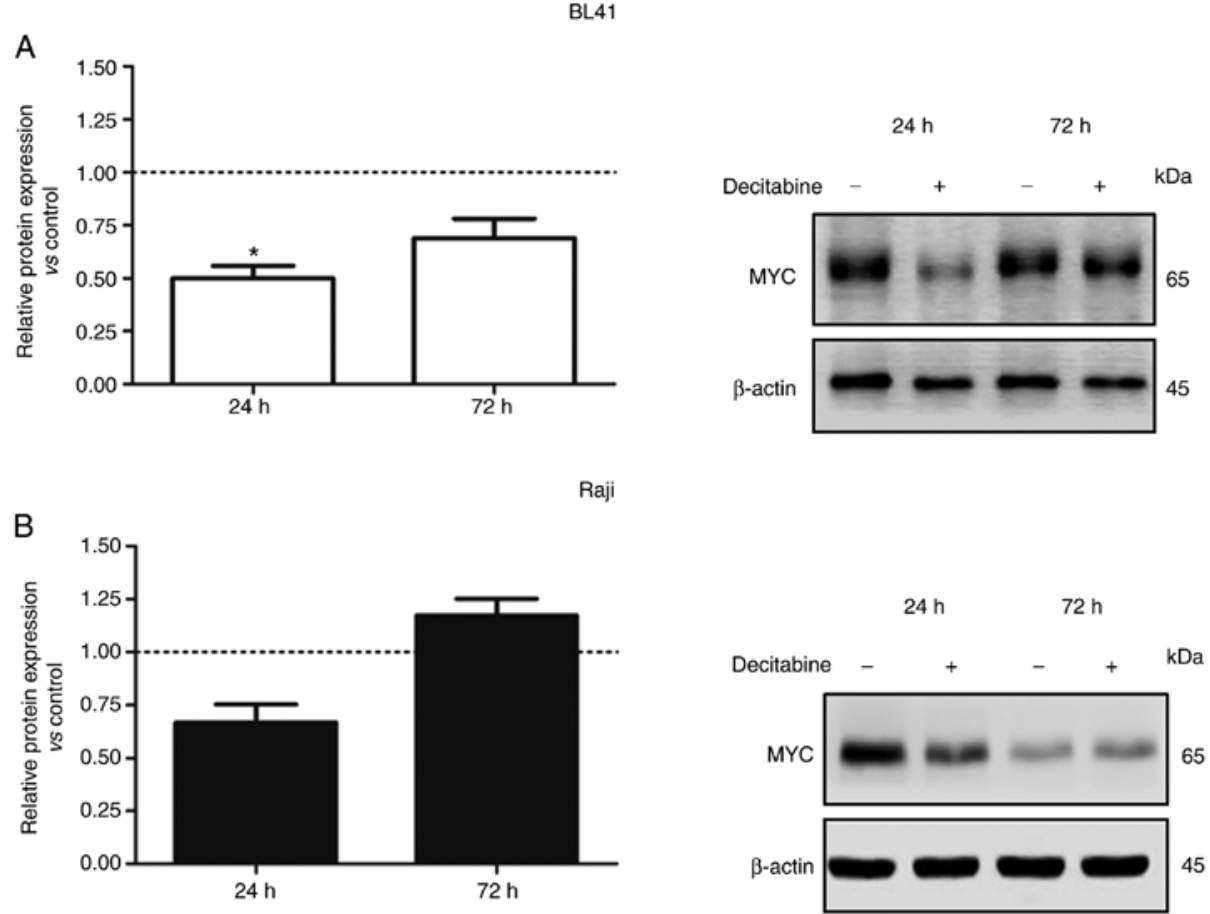

C
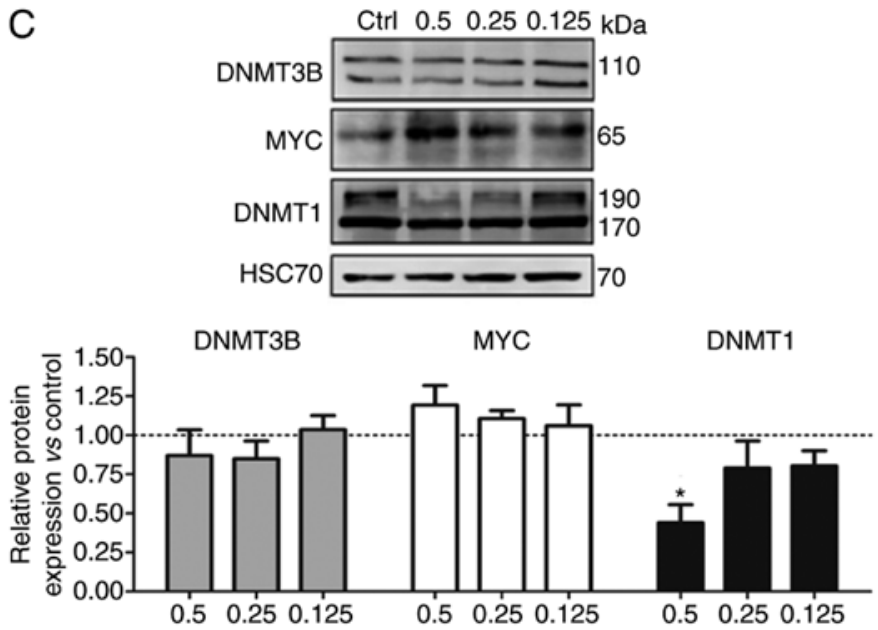

D
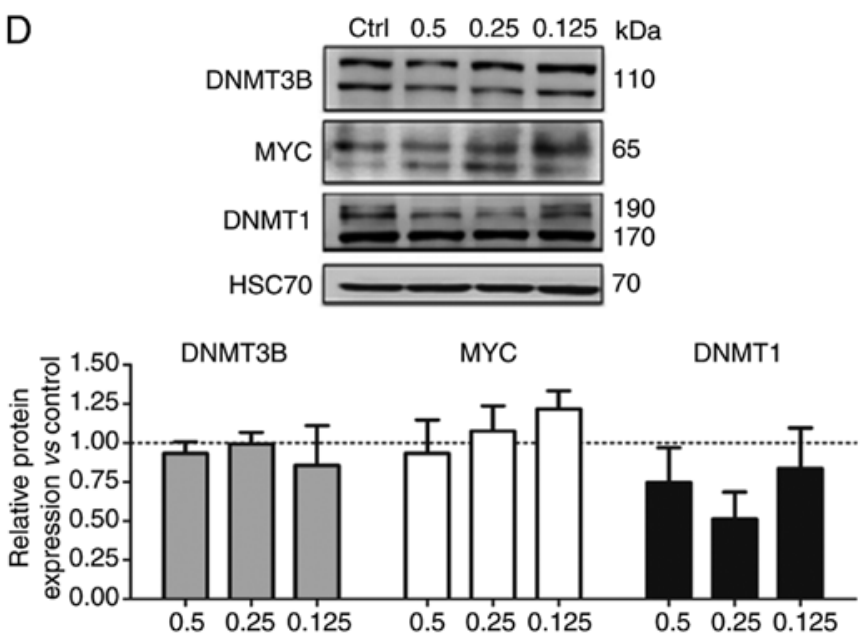

Figure 3. DNA methyltransferase inhibitor decitabine downregulates MYC protein levels in a dose-dependent manner. Western blot analysis of MYC expression after decitabine treatment. Band intensities were assessed by densitometry analysis (left), and a representative western blot image is displayed on the right. (A) BL41 and (B) Raji cells were evaluated at 24 and $72 \mathrm{~h}$ after decitabine treatment $(1.0 \mu \mathrm{M})$. The dotted line represents the untreated control. $\beta$-actin was used as the endogenous control. DNMT1, DNMT3B and MYC protein expression were evaluated by western blotting in BL cell lines (C) BL41 and (D) Raji after treatment with low doses of decitabine $(0.5,0.25$ and $0.125 \mu \mathrm{M})$ for $72 \mathrm{~h}$. HSC70 was used as the endogenous control (upper panels). Band intensities were assessed by densitometry (lower panels). The dotted line represents the untreated control. Data presented are the mean of three independent experiments plus the standard deviation. Statistical analysis was performed using Kruskal-Wallis and Dunnett's post hoc test. "P<0.05. Decitabine, 5-aza-2'-deoxycytidine. 
A

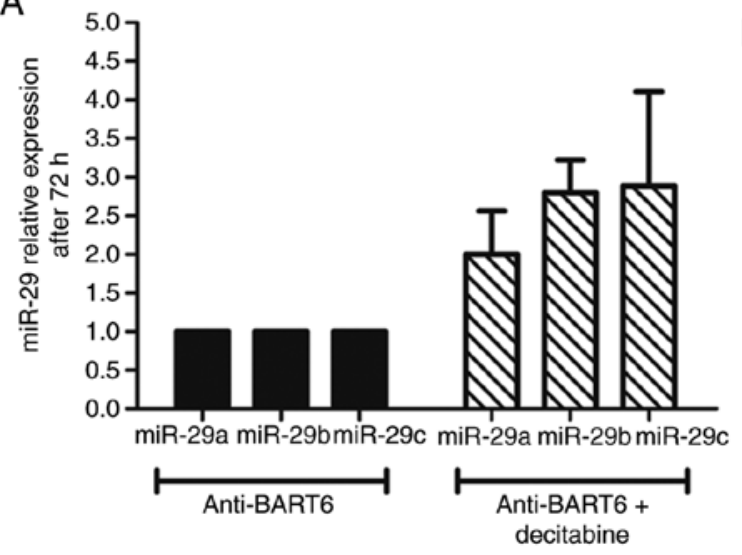

B

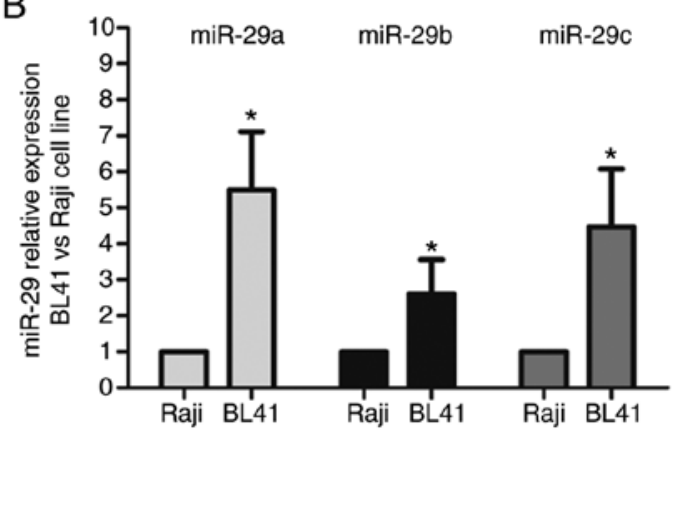

Figure 4. Inhibition of EBV-miR-BART6-5p combined with decitabine treatment enhances miR-29 expression in an EBV-BL cell line. miR-29 expression was evaluated by real-time quantitative PCR in BL41 and Raji cells. (A) Raji cells were transfected with the antagomir for BART6-5p, followed by decitabine $(1.0 \mu \mathrm{M})$ treatment, and harvested $72 \mathrm{~h}$ later for miR-29 expression analysis. Data from two independent experiments are presented. (B) The relative levels of $\mathrm{miR}-29 \mathrm{a} / \mathrm{b} / \mathrm{c}$ expression between untreated BL41 and untreated Raji cells. The results revealed the fold increases in the miR-29a/b/c expression levels in BL41 cells compared to Raji cells. Data presented are the mean of five independent experiments plus the standard deviation and statistical analysis between Raji and BL41 cells was performed using Kruskal-Wallis and Dunnett's post hoc test. ' $\mathrm{P}<0.05$. Decitabine, 5-aza-2'-deoxycytidine.

that are associated with cell cycle control, proliferation, and apoptosis $(21,23)$. miR-29s were previously reported to be regulated by MYC contributing to tumorigenesis (33). The protein c-MYC has a critical role in different cellular processes and its protein stability is tightly regulated $(34,35)$. In $\mathrm{BL}$, the chromosomal translocation places its promoter next to the immunoglobulin enhancers, increasing the transcription of the mRNA, followed by protein translation (9-11).

MYC promotes B-cell lymphomagenesis through a complex interplay with several signalling pathways, including miRNA regulation (36-39). Among the several MYC-regulated miRNAs, it was revealed that MYC may suppress the expression of miR-29s through the recruitment of the HDAC3/EZH2 co-repressor complex to the promoter region of miR-29 genes (20). EZH2 and HDCA3 are part of the Polycomb repressive complex 2 (PRC2), which specifically trimethylates histones at K27, resulting in epigenetic silencing. Although MYC activity can mediate the suppression of miR-29 through HDAC and EZH2 recruitment, other mechanisms can work together to potentiate suppression. In fact, more than one mechanism of epigenetic regulation can cooperate and enhance regulation (28). Notably, it is worth considering the promoter tissue miRNA-specificity regarding aberrant miRNA cancer methylation $(28,40)$. Approximately $45.5 \%$ of miRNA genes have been shown to be methylated in at least two cancer types (41). Epigenetic silencing by promoter hypermethylation of miRNAs with tumour suppressor activities has been described in different types of cancers, being associated with aberrant miRNA expression $(41,42)$. Therefore, miRNA methylation in cancer has been investigated by pharmacological unmasking experimental assays using a DNA methyltransferase inhibitor, 5-aza-2'-deoxycytidine (5-aza-CdR, DAC or decitabine), and histone deacetylase (HDAC) inhibitors, as well as double knockout models for DNA methyltransferase enzymes DNMT1 and DNMT3B $(43,44)$. Given the regulatory role of MYC in miR-29 expression, methylation was assessed as an additional mechanism that may contribute to miR-29 silencing in BL tumour samples. In the previous study we used MSP assays to reveal the miR-29 promoter and enhancer gene methylation in BL cell lines (21). The MSP assay has known disadvantages such as the use of relative comparisons instead of absolute quantification $(45,46)$. Using quantitative bisulfite pyrosequencing assays, methylation was investigated in $\mathrm{CpG}$ sites located in the promoter and enhancer regions of $\mathrm{miR}-29 \mathrm{a} / \mathrm{b} / \mathrm{c}$ in BL tumour samples. It was demonstrated that BL cell lines as well as BL tumours exhibit hypermethylation of $m i R-29 a / b 1$ enhancer and $m i R-29 b 2 / c$ promoter regions.

It has been proposed that the determination of cell identity during development is highly associated with enhancer usage, which is locally regulated by DNA methylation (47). Active enhancers are characterized by a higher density of transcription factor binding sites, DNA hypomethylation, a high level of H3K27ac and the binding of a mediator (48). However, enhancers not only play a role in early lineage commitment but also regulate the expression of cell type-specific genes in differentiated cells, such as macrophages and B cells (48). Other key determinants of cell fate include transcription factors and miRNAs, enclosing a complex regulatory network where these different factors interact with each other (5). It is not surprising that these networks are often dysregulated in cancer, a condition in which cell identity and commitment to tissue homeostasis is lost. Notably, differential expression of key transcription factors, such as MYC overexpression in BL (9-11), of miRNAs, as exemplified by the upregulation of miR-21 and downregulation of let-7 in breast cancer (49), and alterations in enhancer methylation have been revealed in tumours (50). In the present study, we propose a complex network that may play a role in BL pathogenesis, in which MYC overexpression, a common feature of this neoplasia, leads to DNMT1 and DNMT3B upregulation that is associated with miR-29 silencing by methylation of promoter and enhancer regions.

The MYC/miR-29/DNMT3B circuit was identified by El Baroudi et al as a feed-forward loop in which a master transcription factor regulates a miRNA and, together with it, 
a set of protein coding genes that are targeted by the same miRNA (Type II) (51). Moreover, these feedback regulations point to complex networks between miRNAs and epigenetic machinery enhancing the human gene regulatory network. Notably, MYC can lead to epigenetic deregulation through induction of DNMT1 and DNMT3B transcription. The transcriptional regulation of DNMT3B by MYC during B-cell lymphomagenesis has been recently demonstrated in vivo (52). It is noteworthy to mention that a previous study of our group demonstrated overexpression of DNMT3B in $~ 86 \%$ of BL tumour samples (23). Additionally, it was also described by Poole et al that MYC binds to the DNMT1 and DNMT3B promoters in BL-like cells and deregulates the expression of both DNMT1 and DNMT3B. Moreover, the knockdown of endogenous MYC leads to diminished DNMT3B expression levels in human BL cell lines. Thus, a model was proposed where MYC additionally controls DNA methylation through overexpression of DNMT1 and DNMT3B during lymphomagenesis (53).

It has also previously revealed that $\mathrm{miR}-29 \mathrm{a} / \mathrm{b} / \mathrm{c}$ expression is downregulated in BL tumour samples (23). Since miRNA expression can be lost due to methylation, as demonstrated in the present study in BL cell lines, $10 \mathrm{BL}$ tumour samples were screened for methylation on miR-29a/b1 and $\mathrm{miR}-29 \mathrm{~b} 2 / \mathrm{c}$. Methylation in CpG sites located in the promoter and enhancer regions was also confirmed in tumour tissues. These findings suggest a relationship between downregulation of tumour-suppressive miR-29a/b/c, overexpression of DNMT1 and DNMT3B and hypermethylation in miR-29a/b1 and $m i R-29 b 2 / c$ in BL cells.

Another layer of complexity in miR-29 regulation was revealed in our study using an EBV-positive BL cell line (Raji). Regarding methylation of the promoter, promoter flanking region, and enhancer regions of miR-29, it was observed in the Raji cells that although treatment with lower concentrations of decitabine decreased the methylation levels of evaluated CpG sites, it did not increase miR-29 expression. One possible explanation would be related to EBV, which has been reported to decrease the levels of miRNAs including miR-29 in peripheral blood-derived B-cells infected in vitro with EBV (54). EBV proteins have also been reported to contribute to methylation, in which the EBV proteins EBNA3A/C bind next to the $B I M$ gene promoter, followed by $\mathrm{CpG}$ methylation (55). Although EBV latency may vary among BL EBV-positive cell lines, miRNA production is a common feature among the different EBV latency types (56). Amongst miRNAs, the EBV-BART cluster comprised of several miRNAs has been revealed to regulate both EBV and host protein expression (57). It has been reported that EBV-miRNA-BART prevents apoptosis by inhibiting caspase- 3 and enhances cell growth of EBV-infected B-cells. Thus, EBV maintains lymphomas via its miRNAs (58). Notably, BART6 decreases DICER1 expression, which is involved in miRNA maturation in the cell cytoplasm (27). In the present study, after transfection with an antagomir to specifically inhibit BART6-5p, the treatment with decitabine resulted in increased miR-29 expression. The results indicated a role for EBV miRNAs in the complex miR-29 regulation of BL tumours associated with EBV adding a new layer of complexity in miR-29 regulation in BL tumours.
Furthermore, it was demonstrated for the first time by pyrosequencing of multiple $\mathrm{CpG}$ sites that $\mathrm{BL}$ tumour samples present high levels of methylation in both promoter and enhancer regions. Although the idea of a functional assay to evaluate the activity of the enhancer would be relevant, Suzuki et al (5) have shown that the region analysed by us in miR-29a/b1 sequence (chr7: 130,582,141-130,582,237, genome version GRCh37/hg19) flanks a typical enhancer region (chr7:130582783-130585367, genome version GRCh37/hg19) identified in CD19-positive primary cells, and encompasses a super-enhancer region (chr7: 130577042-130754905, genome version GRCh37/hg19) in CD20-positive cells. The same authors have also revealed that enhancers and super-enhancers are cell type-specific and their activity has a great impact on cell fate, which could be mediated by the regulation of master miRNAs. Therefore, the miR-29a/b1 region analysed in our study is considered as specifically relevant for our model on BL.

In summary, the miR-29a/b1 and miR-29b2/c genes have methylated $\mathrm{CpG}$ sequences at promoter and enhancer regions that may contribute to the regulation of the expression of miR-29s in BL tumours. The findings indicate interplay between MYC and miR-29 regulation, highlighting the potential role of EBV-microRNAs in miR-29 regulation for BL pathogenesis. On the other hand, studies focusing on EBV-miRNAs in lymphomas are limited to cell lines. Animal models for EBV-associated carcinogenesis are required to address the regulatory network involving EBV-miRNAs.

\section{Acknowledgements}

Not applicable.

\section{Funding}

INCT for Cancer Control: CNPq 573806/2008-0/FAPERJE26/ 170.026/2008; FAPERJ E-26/110.375/2014; SWISS-BRIDGE Foundation, sub-project 1B/2014-2018. LM and MCR were supported by grants from the Brazilian National Cancer Institute (INCA), Health Ministry.

\section{Availability of data and materials}

The datasets generating during this study are included within the article; data not shown is available from the corresponding author upon request.

\section{Authors' contributions}

CEK and LM devised the study concept. LM and MCR performed the experiments and generated and analysed the data. LM, MCR, SCSL, and CEK interpreted the data and drafted the manuscript. CEB revised the BL cases and performed FISH assays. CEK and SCSl obtained funding and supervised the study. All authors critically reviewed and approved the final version of the manuscript and agree to be accountable for all aspects of the research in ensuring that the accuracy or integrity of any part of the work are appropriately investigated and resolved. 


\section{Ethics approval and consent to participate}

The present study was approved by the Brazilian National Cancer Institute Ethics Committee (registration number 18/09) in accordance with the Declaration of Helsinki.

\section{Patient consent for publication}

Not applicable.

\section{Competing interests}

The authors declare that that they have no competing interests.

\section{References}

1. Esteller M: Epigenetic changes in cancer. F1000 Biol Rep 3: 9, 2011.

2. Bartel DP: MicroRNAs: Target recognition and regulatory functions. Cell 136: 215-233, 2009.

3. Ying SY, Chang CP and Lin SL: Intron-mediated RNA interference, intronic microRNAs, and applications. Methods Mol Biol 629: 205-237, 2010.

4. Moutinho C and Esteller M: MicroRNAs and epigenetics. Adv Cancer Res 135: 189-220, 2017.

5. Suzuki HI, Young RA and Sharp PA: Super-enhancer-mediated RNA processing revealed by integrative MicroRNA network analysis. Cell 168: 1000-1014.e15, 2017.

6. Landgraf P, Rusu M, Sheridan R, Sewer A, Iovino N, Aravin A, Pfeffer S, Rice A, Kamphorst AO, Landthaler M, et al: A mammalian microRNA expression atlas based on small RNA library sequencing. Cell 129: 1401-1414, 2007.

7. Hochberg J, Waxman IM, Kelly KM, Morris E and Cairo MS: Adolescent non-Hodgkin lymphoma and Hodgkin lymphoma: State of the science. Br J Haematol 144: 24-40, 2009.

8. Brady G, Macarthur GJ and Farrell PJ: Epstein-Barr virus and Burkitt lymphoma. Postgrad Med J 84: 372-377, 2008.

9. Dalla-Favera R, Bregni M, Erikson J, Patterson D, Gallo RC and Croce CM: Human c-myc onc gene is located on the region of chromosome 8 that is translocated in Burkitt lymphoma cells. Proc Natl Acad Sci USA 79: 7824-7827, 1982.

10. Taub R, Kirsch I, Morton C, Lenoir G, Swan D, Tronick S, Aaronson S and Leder P: Translocation of the c-myc gene into the immunoglobulin heavy chain locus in human Burkitt lymphoma and murine plasmacytoma cells. Proc Natl Acad Sci USA 79: 7837-7841, 1982.

11. Willis TG and Dyer MJ: The role of immunoglobulin translocations in the pathogenesis of B-cell malignancies. Blood 96: 808-822, 2000.

12. Schmitz R, Young RM, Ceribelli M, Jhavar S, Xiao W, Zhang M, Wright G, Shaffer AL, Hodson DJ, Buras E, et al: Burkitt lymphoma pathogenesis and therapeutic targets from structural and functional genomics. Nature 490: 116-120, 2012.

13. Sander S, Calado DP, Srinivasan L, Köchert K, Zhang B, Rosolowski M, Rodig SJ, Holzmann K, Stilgenbauer S, Siebert R, et al: Synergy between PI3K signaling and MYC in Burkitt lymphomagenesis. Cancer Cell 22: 167-179, 2012.

14. Oduor CI, Kaymaz Y, Chelimo K, Otieno JA, Ong'echa JM, Moormann AM and Bailey JA: Integrative microRNA and mRNA deep-sequencing expression profiling in endemic Burkitt lymphoma. BMC Cancer 17: 761, 2017.

15. Lenze D, Leoncini L, Hummel M, Volinia S, Liu CG, Amato T, De Falco G, Githanga J, Horn H, Nyagol J, et al: The different epidemiologic subtypes of Burkitt lymphoma share a homogenous micro RNA profile distinct from diffuse large B-cell lymphoma. Leukemia 25: 1869-1876, 2011.

16. Hezaveh K, Kloetgen A, Bernhart SH, Mahapatra KD, Lenze D, Richter J, Haake A, Bergmann AK, Brors B, Burkhardt B, et al: Alterations of microRNA and microRNA-regulated messenger RNA expression in germinal center B-cell lymphomas determined by integrative sequencing analysis. Haematologica 101: 1380-1389, 2016.

17. Zhu K, Liu L, Zhang J, Wang Y, Liang H, Fan G, Jiang Z, Zhang CY, Chen X and Zhou G: miR-29b suppresses the proliferation and migration of osteosarcoma cells by targeting CDK6. Protein Cell 7: 434-444, 2016.
18. Kwon JJ, Factora TD, Dey S and Kota J: A systematic review of miR-29 in cancer. Mol Ther Oncolytics 12: 173-194, 2019.

19. Garzon R, Heaphy CE, Havelange V, Fabbri M, Volinia S, Tsao T, Zanesi N, Kornblau SM, Marcucci G, Calin GA, et al: MicroRNA 29b functions in acute myeloid leukemia. Blood 114: 5331-5341, 2009.

20. Zhang X, Zhao X, Fiskus W, Lin J, Lwin T, Rao R, Zhang Y, Chan JC, Fu K, Marquez VE, et al: Coordinated silencing of MYC-mediated miR-29 by HDAC3 and EZH2 as a therapeutic target of histone modification in aggressive B-Cell lymphomas. Cancer Cell 22: 506-523, 2012.

21. Mazzoccoli L, Robaina MC, Apa AG, Bonamino M, Pinto LW, Queiroga E, Bacchi CE and Klumb CE: miR-29 silencing modulates the expression of target genes related to proliferation, apoptosis and methylation in Burkitt lymphoma cells. J Cancer Res Clin Oncol 144: 483-497, 2018.

22. Campo E, Swerdlow SH, Harris NL, Pileri S, Stein H and Jaffe ES: The 2008 WHO classification of lymphoid neoplasms and beyond: Evolving concepts and practical applications. Blood 117: 5019-5032, 2011.

23. Robaina MC, Mazzoccoli L, Arruda VO, Reis FR, Apa AG, de Rezende LM and Klumb CE: Deregulation of DNMT1, DNMT3B and miR-29s in Burkitt lymphoma suggests novel contribution for disease pathogenesis. Exp Mol Pathol 98: 200-207, 2015 .

24. Livak KJ and Schmittgen TD: Analysis of relative gene expression data using real-time quantitative PCR and the 2(-Delta Delta C(T)) method. Methods 25: 402-408, 2001

25. Guan H, Xie L, Klapproth K, Weitzer CD, Wirth T and Ushmorov A: Decitabine represses translocated MYC oncogene in Burkitt lymphoma. J Pathol 229: 775-783, 2013.

26. Shinozaki-Ushiku A, Kunita A, Isogai M, Hibiya T, Ushiku T, Takada K and Fukayama M: Profiling of virus-encoded MicroRNAs in Epstein-Barr Virus-associated gastric carcinoma and their roles in gastric carcinogenesis. J Virol 89: 5581-5591, 2015.

27. Iizasa H, Wulff BE, Alla NR, Maragkakis M, Megraw M, Hatzigeorgiou A, Iwakiri D, Takada K, Wiedmer A, Showe L, et al: Editing of Epstein-Barr virus-encoded BART6 microR NAs controls their dicer targeting and consequently affects viral latency. J Biol Chem 285: 33358-33370, 2010 .

28. Malumbres M: miRNAs and cancer: An epigenetics view. Mol Aspects Med 34: 863-874, 2013.

29. Fabbri M, Ivan M, Cimmino A, Negrini $M$ and Calin GA: Regulatory mechanisms of microRNAs involvement in cancer. Expert Opin Biol Ther 7: 1009-1019, 2007.

30. Zhao JJ, Lin J, Lwin T, Yang H, Guo J, Kong W, Dessureault S, Moscinski LC, Rezania D, Dalton WS, et al: microRNA expression profile and identification of miR-29 as a prognostic marker and pathogenetic factor by targeting CDK6 in mantle cell lymphoma. Blood 115: 2630-2639, 2010.

31. Kinoshita T, Nohata N, Hanazawa T, Kikkawa N, Yamamoto N, Yoshino H, Itesako T, Enokida H, Nakagawa M, Okamoto Y and Seki N: Tumour-suppressive microRNA-29s inhibit cancer cell migration and invasion by targeting laminin-integrin signalling in head and neck squamous cell carcinoma. Br J Cancer 109: 2636-2645, 2013

32. Nishikawa R, Chiyomaru T, Enokida H, Inoguchi S, Ishihara T, Matsushita R, Goto Y, Fukumoto I, Nakagawa M and Seki N: Tumour-suppressive microRNA-29s directly regulate LOXL2 expression and inhibit cancer cell migration and invasion in renal cell carcinoma. FEBS Lett 589: 2136-2145, 2015.

33. Chang TC, Yu D, Lee YS, Wentzel EA, Arking DE, West KM, Dang CV, Thomas-Tikhonenko A and Mendell JT: Widespread microRNA repression by Myc contributes to tumorigenesis. Nat Genet 40: 43-50, 2008.

34. Sears RC: The life cycle of C-myc: From synthesis to degradation. Cell Cycle 3: 1133-1137, 2004.

35. Majello B and Perini G: Myc proteins in cell biology and pathology. Biochim Biophys Acta 1849: 467-468, 2015.

36. Zeller KI, Zhao X, Lee CW, Chiu KP, Yao F, Yustein JT, Ooi HS, Orlov YL, Shahab A, Yong HC, et al: Global mapping of c-Myc binding sites and target gene networks in human B cells. Proc Natl Acad Sci USA 103: 17834-17839, 2006.

37. Meyer N and Penn LZ: Reflecting on 25 years with MYC. Nat Rev Cancer 8: 976-990, 2008.

38. Kim JW, Mori S and Nevins JR: Myc-induced microRNAs integrate Myc-mediated cell proliferation and cell fate. Cancer Res 70: 4820-4828, 2010 . 
39. Tao J, Zhao X and Tao J: c-MYC-miRNA circuitry: A central regulator of aggressive $\mathrm{B}$-cell malignancies. Cell Cycle 13: 191-198, 2014.

40. Baer C, Claus R and Plass C: Genome-wide epigenetic regulation of miRNAs in cancer. Cancer Res 73: 473-477, 2013.

41. Strmsek Z and Kunej T: MicroRNA silencing by DNA methylation in human cancer: A literature analysis. Noncoding RNA 1: 44-52, 2015

42. Lopez-Serra P and Esteller M: DNA methylation-associated silencing of tumor-suppressor microRNAs in cancer. Oncogene 31: 1609-1622, 2012.

43. Lujambio A and Esteller M: CpG island hypermethylation of tumor suppressor microRNAs in human cancer. Cell Cycle 6 : $1455-1459,2007$.

44. Saito Y and Jones PA: Epigenetic activation of tumor suppressor microRNAs in human cancer cells. Cell Cycle 5: 2220-2222, 2006.

45. Tost J and Gut IG: DNA methylation analysis by pyrosequencing. Nat Protoc 2: 2265-2275, 2007.

46. Colella S, Shen L, Baggerly KA, Issa JP and Krahe R: Sensitive and quantitative universal Pyrosequencing methylation analysis of CpG sites. Biotechniques 35: 146-150, 2003.

47. Perino $\mathrm{M}$ and Veenstra GJ: Chromatin control of developmental dynamics and plasticity. Dev Cell 38: 610-620, 2016.

48. Whyte WA, Orlando DA, Hnisz D, Abraham BJ, Lin CY, Kagey MH, Rahl PB, Lee TI and Young RA: Master transcription factors and mediator establish super-enhancers at key cell identity genes. Cell 153: 307-319, 2013.

49. Elghoroury EA, ElDine HG, Kamel SA, Abdelrahman AH, Mohammed A, Kamel MM and Ibrahim MH: Evaluation of miRNA-21 and miRNA let-7 as prognostic markers in patients with breast cancer. Clin Breast Cancer 18: e721-e726, 2018.
50. Karpinski P, Pesz K and Sasiadek MM: Pan-cancer analysis reveals presence of pronounced DNA methylation drift in CpG island methylator phenotype clusters. Epigenomics 9: 1341-1352, 2017.

51. El Baroudi M, Corà D, Bosia C, Osella M and Caselle M: A curated database of miRNA mediated feed-forward loops involving MYC as master regulator. PLoS One 6: e14742, 2011.

52. Sabò A, Kress TR, Pelizzola M, De Pretis S, Gorski MM, Tesi A Morelli MJ, Bora P, Doni M, Verrecchia A, et al: Selective transcriptional regulation by Myc in cellular growth control and lymphomagenesis. Nature 511: 488-492, 2014.

53. Poole CJ, Zheng W, Lodh A, Yevtodiyenko A, Liefwalker D, $\mathrm{Li} \mathrm{H}$, Felsher DW and van Riggelen J: DNMT3B overexpression contributes to aberrant DNA methylation and MYC-driven tumor maintenance in T-ALL and Burkitt's lymphoma. Oncotarget 8: 76898-76920, 2017.

54. Godshalk SE, Bhaduri-McIntosh S and Slack FJ: Epstein-Barr virus-mediated dysregulation of human microRNA expression. Cell Cycle 7: 3595-3600, 2008.

55. Paschos K, Smith P, Anderton E, Middeldorp JM, White RE and Allday MJ: Epstein-barr virus latency in B cells leads to epigenetic repression and $\mathrm{CpG}$ methylation of the tumour suppressor gene Bim. PLoS Pathog 5: e1000492, 2009.

56. Price AM and Luftig MA: To be or not IIb: A multi-step process for Epstein-Barr virus latency establishment and consequences for B cell tumorigenesis. PLoS Pathog 11: e1004656, 2015.

57. Klinke O, Feederle R and Delecluse HJ: Genetics of Epstein-Barr virus microRNAs. Semin Cancer Biol 26: 52-59, 2014.

58. Vereide DT, Seto E, Chiu YF, Hayes M, Tagawa T, Grundhoff A, Hammerschmidt W and Sugden B: Epstein-Barr virus maintains lymphomas via its miRNAs. Oncogene 33: 1258-1264, 2014. 